\title{
DNA/Lipid Complex Incorporated with Fibronectin to Cell Adhesion Enhances Transfection Efficiency in Prostate Cancer Cells and Xenografts
}

\author{
Yoshiyuki Hattori and Yoshie Maitani* \\ Institute of Medicinal Chemistry, Hoshi University; 2-4-41 Ebara, Shinagawa-ku, Tokyo 142-8501, Japan. \\ Received August 19, 2006; accepted December 20, 2006; published online December 22, 2006
}

\begin{abstract}
Previously we have described the development and applications of lipid-based nanoparticles for gene delivery vector. In an attempt to improve transfection efficiency using the cell adhesion of extracellular matrix (ECM) to DNA/lipid complex (nanoplex), the mRNA expression of integrin $\alpha 2 \beta 1$ and CD44 in prostate cancer cells was detected as adhesion molecules for fibronectin (Fn), collagen I (Col) and laminin (Lam) using a commercially available cDNA array (GEArray ${ }^{\mathrm{TM}}$ ) system. These ECM proteins could enhance DNA transfection activity in cells when coated on the nanoplex. Among the ECM proteins, Fn-coating nanoplexes significantly increased transfection activity 2-fold in prostate cancer PC-3 cells, and exhibited higher DNA transfection activities to PC3 xenografts, compared with commercially available cationic polymer in vivo jetPEI. These results indicated that Fn-coating nanoplexes could facilitate efficient transfection of prostate tumor cells.
\end{abstract}

Key words adhesion molecule; prostate tumor; nanoparticle; transfection; fibronectin; collagen I

Extracellular matrix (ECM) consists of a group of wellcharacterized glycoproteins, which are present at the epithelial-mesenchymal interface of both begin and malignant tissues. ${ }^{1)}$ Tumor cell adhesion to ECM components such as fibronectin (Fn), laminin (Lam) and collagen 1 (Col) is mediated via integrin subunits, and ECM components play a major role in the invasion and metastasis of tumor cells. ${ }^{2)}$

ECM protein and adhesion molecules have been used for tumor gene delivery. The arginin-glycine-aspartic acid (RGD) sequence is well known to serve as a recognition motif in multiple ligands for several different integrins such as integrin $\alpha \mathrm{v} \beta 3$ and $\alpha 5 \beta 1$ et al. ${ }^{3)}$ RGD-modified liposomes ${ }^{4,5)}$ and polymers ${ }^{6,7)}$ have been developed as gene transfer vectors. An electrostatic complex consisting of a cationic liposome, an integrin $\alpha 5 \beta 1$-targeting peptide, and plasmid DNA, was efficiently vector transfected to tumor cells. ${ }^{8,9)} \mathrm{Re}-$ cently, it has been reported that calcium phosphate/DNA complex with ECM proteins, especially $\mathrm{Col}$ and Fn, led to remarkably high transgene expression in mammalian cells. ${ }^{10}$ ) Cells can attach to ECM proteins via plasma membrane receptor proteins. Therefore, ECM proteins for adhesion to tumor cells can be useful for gene delivery to increase gene expression by enhancing the association of genes with cells. However, no attention was paid to the complexation of lipidbased cationic liposomes or nanoparticles with ECM proteins.

Lipid-based cationic nanoparticles could deliver DNA with significantly high transfection efficiency into prostate tumor cells. ${ }^{11)}$ To improve nanoparticle-mediated transfection for in vivo application, the effect of cell adhesion of nanoplex on gene transfer efficiency was investigated. First, mRNA expression of integrin $\alpha 2 \beta 1$ and CD44 in prostate cancer cells was detected as adhesion molecules for Fn, Col and Lam using a commercially available cDNA array $\left(\right.$ GEArray $\left.{ }^{\mathrm{TM}}\right)$ system. Second, we prepared a ternary complex of the nanoparticle/DNA complex (nanoplex) with Fn, Col and Lam (Fn, Col and Lam nanoplex) and evaluated transfection activity in PC-3 cells and xenografts.

\section{MATERIALS AND METHODS}

Cell Culture Prostate tumor LNCaP and PC-3 cells were supplied by the Cell Resource Center for Biomedical Research, Tohoku University (Miyagi, Japan). The cells were grown in a RPMI-1640 medium (Life Technologies, Inc., Grand Island, NY, U.S.A.) supplemented with $10 \%$ heat-inactivated fetal bovine serum (FBS) (Life Technologies, Inc.) and kanamycin $(100 \mu \mathrm{g} / \mathrm{ml})$ at $37^{\circ} \mathrm{C}$ in a $5 \% \mathrm{CO}_{2}$ humidified atmosphere.

Cell Spreading For coating Fn, Col and Lam onto tissue culture dishes, Fn (human fibronectin, BD Bioscience, Bedford, MA, U.S.A.), Col (human collagen I, Morinaga Institute of Biological Science, Yokohama, Japan) and Lam (mouse laminin, Harbor Bio-Products, Norwood, MA, U.S.A.), respectively, were diluted to $50 \mu \mathrm{g} / \mathrm{ml}$ with PBS (pH 7.4), and added to $35 \mathrm{~mm}$ tissue culture dishes. The plates were incubated for $1 \mathrm{~h}$ at $37^{\circ} \mathrm{C}$. After coating the plates, they were washed 3 times in PBS, and were plated $1 \times 10^{6} \mathrm{PC}-3$ or LNCaP cells in RPMI-1640 medium onto the dishes. After $3 \mathrm{~h}$ incubation, the unattached cells were removed by exchanging the medium, and the attached cells on the dishes were examined microscopically for the number of spread cells.

cDNA Array Total RNA from LNCaP or PC-3 cells was extracted using the RNasy mini kit (Qiagen, Hilden, Germany). Non-radioactive human extracellular matrix and an adhesion molecule gene array (Super Array, Inc., MD, U.S.A.) were used to analyze the gene expression profile of extracellular matrix and adhesion molecules according to the manufacturer's protocol. The results were analyzed using free ScanAlyze software (developed by Dr. Michael Eisen), which converts a grayscale TIFF image of spots into numerical data (median pixel intensity), and then the gene expression profiles were compared using GEArray analyzer software (Super Array, Inc.). Normalized intensities were calculated from each array by subtracting the negative control from each spot.

Preparation of Plasmid DNA The plasmid pCMV-luc encoding the luciferase gene under the control of the CMV 
promoter was constructed as previously described. ${ }^{12)}$ A protein-free preparation of these plasmids was purified following alkaline lysis using maxiprep columns (Qiagen).

Preparation and Size Measurement of Nanoparticles and Nanoplexes Cholesteryl- $3 \beta$-carboxyamidoethylene- $N$ hydroxyethylamine (OH-Chol) was synthesized as previously described. ${ }^{11)}$ Tween 80 was obtained from NOF Co., Ltd. (Tokyo, Japan). Lipid-based nanoparticles were prepared with lipids $(\mathrm{OH}-\mathrm{Chol}$ : Tween $80=10: 1.3 \mathrm{mg})$ in $10 \mathrm{ml}$ water by the modified ethanol injection method as previously described. ${ }^{11)}$ The nanoplex at a charge ratio $(+/-)$ of cationic lipid to DNA of 3/1 was formed by the addition of $9.5 \mu \mathrm{l}$ of the nanoparticle solution to $2 \mu \mathrm{g}$ DNA in $50 \mu \mathrm{l}$ of $50 \mathrm{~mm}$ $\mathrm{NaCl}$ solution with gentle shaking and leaving at room temperature for $10 \mathrm{~min}$. To prepare ternary complexes with ECM protein (Fn, Col and Lam nanoplex), the nanoplex with $2 \mu \mathrm{g}$ DNA was mixed with 2 or $5 \mu \mathrm{g}$ of Fn, Col and Lam, respectively, and incubated for $10 \mathrm{~min}$. The particle size distributions and $\zeta$-potentials were measured by the dynamic light scattering method and the electrophoresis light scattering method, respectively (ELS-800, Otsuka Electronics Co., Ltd., Osaka, Japan), at $25^{\circ} \mathrm{C}$ after the dispersion was diluted to an appropriate volume with water.

Luciferase Assay For transfection, Fn, Col and Lam nanoplexes, respectively, were diluted in $1 \mathrm{ml}$ of medium supplemented with 10\% FBS and then incubated for $24 \mathrm{~h}$. Lipofectamine 2000 (Invitrogen Corp., Carlsbad, CA, U.S.A.) lipoplex at a charge ratio $(+/-)$ of $2 / 1$ of cationic lipid to DNA was prepared according to the manufacturer's protocol. Luciferase expression was measured as counts per second (cps) $/ \mu$ g protein using the luciferase assay system (Pica gene, Toyo Ink Mfg. Co., Ltd., Tokyo, Japan) and BCA reagent (Pierce, Rockford, IL, U.S.A.) as previously reported. ${ }^{13)}$

Cell Viability The cell viabilities upon transfection using Fn, Col and Lam nanoplex were evaluated with a cell proliferation assay kit (Dojindo, Kumamoto, Japan). Cells were placed in a 96-well plate in the medium containing serum, and were transfected with nanoplex of $0.2 \mu \mathrm{g}$ plasmid DNA with ECM protein $(0.2 \mu \mathrm{g})$. After $24 \mathrm{~h}$ of incubation, the cell viabilities were measured according to the manufacturer's protocol.

In Vivo Transfection To generate PC-3 tumor xenografts, $1 \times 10^{7}$ cells suspended in $50 \mu \mathrm{l}$ of RPMI medium were inoculated subcutaneously into the flank of male BALB/c nu/nu mice ( 7 weeks of age, CLEA Japan, Inc., Tokyo, Japan). To prepare Fn nanoplex, the nanoplex with $10 \mu \mathrm{g}$ DNA at a charge ratio $(+/-)$ of cationic lipid to DNA of $3 / 1$ was mixed with $10 \mu \mathrm{g}$ of Fn as described above section. The Fn nanoplex was administered to the xenografts with a tumor volume of about $200-300 \mathrm{~mm}^{3}$ by intratumoral injection (10 $\mu \mathrm{g}$ of plasmid DNA/tumor) as previously reported. ${ }^{14)}$ In vivo jetPEI, a commercially available cationic polymer transfection reagent (PolyPlus-transfection, Llkirch, France), was used according to the manufacturer's protocol for in vivo administration. Twenty-four hours after injection, D-luciferin (potassium salt) dissolved in PBS $(125 \mathrm{mg} / \mathrm{kg}$ of body weight) was injected into the mouse peritoneal cavity (i.p.) and subsequently anesthetized by i.m. injection of $50 \mathrm{mg} / \mathrm{kg}$ body weight of pentobarbital (Nembutal, Dainippon Pharmaceutical Co., Ltd., Osaka, Japan). In vivo bioluminescence imaging was performed using an NightOWL LB981 NC100 system (Berthold Technologies, Bad Wildbad, Germany). A gray scale body-surface reference image was collected using a NightOWL LB981 CCD camera. Photons emitted from luciferase within the mice and transmitted through its tissues were collected and integrated for a 2 min period. Overlay of the real image and luminescence representation allowed the localization and measurement of luminescence emitted from xenografts. The signal intensities from manually derived regions (ROI) of interest were obtained and data were expressed as photon flux (count/s). Background photon flux was defined from an ROI of the same size placed in a nonluminescent area near the mice and then subtracted from the measured luminescent signal intensity.

Statistical Analysis Data were compared using analysis of variance and evaluated by Student's $t$ test or Welch's $t$ test. A $p$ value of $\leq 0.05$ was considered significant.

\section{RESULTS AND DISCUSSION}

Previously we reported that a lipid-based nanoparticle composed of $\mathrm{OH}-\mathrm{Chol}$ as a cationic lipid showed significantly high transfection efficiency into prostate tumor cells when complexed with plasmid DNA in the presence of $50 \mathrm{~mm} \mathrm{NaCl}$ solution. ${ }^{11)}$ In this study, we improved this nanoparticle by coating ECM proteins onto nanoplex to enhance transfection activity into $\mathrm{PC}-3$ and $\mathrm{LNCaP}$ cells. LNCaP and PC-3 are prostate cancer cell lines with low and high metastatic potential, respectively. ${ }^{15)}$

First, we tested three different ECM proteins for their cell adhesion to a plastic surface. In PC-3 cells, cell adhesion was increased by coating with Fn, Col and Lam (Fig. 1A), but in LNCaP cells, it was only slightly increased by coating with Fn (Fig. 1B). These results indicated that ECM proteins could strongly associate with adhesion molecules on the cell surface of high metastatic PC-3 cells compared with low metastatic LNCaP cells.

To identify which adhesion molecule on the cell surface of a prostate tumor promoted adhesion with ECM protein-coating plates, we utilized a commercially available ECM and adhesion molecule-specific cDNA array (GEArray ${ }^{\mathrm{TM}}$ ) system for the analysis of 96 genes (Table 1). In PC-3 cells, strong mRNA expression was observed for 2 genes, CD44 and catenin $\beta 1$, moderate mRNA expression for 4 genes, integrin $\beta 1$, hyaluronidases, PECAM-1 and maspin, weak mRNA expression for 7 genes, Meth, E-cadherin, CEA, catenin delta 1 , cathepsin $\mathrm{L}$, fibronectin 1 and integrin $\alpha 2$. In $\mathrm{LNCaP}$ cells, strong mRNA expression was observed for 5 genes, Meth, CEA, catenin $\beta 1$, catenin delta 1 and hyaluronidases, moderate mRNA expression for 2 genes, E-cadherin and integrin $\beta 1$, and weak mRNA expression for 3 genes, cathepsin $\mathrm{L}$, fibronectin 1 and maspin. We found the mRNA expression of CD44 and integrin $\alpha 2 \beta 1$ for the receptors of Fn, Col or Lam in PC-3 cells, but not in LNCaP cells. These expression profiles in genes corresponded with previous studies ${ }^{16,17)}$ and confirmed that the adhesion of PC-3 cells to the tissue culture plate was increased by coating with ECM proteins than that of LNCaP cells (Fig. 1).

Next, we prepared nanoplex coating with ECM proteins and evaluated transfection activity into $\mathrm{PC}-3$ and $\mathrm{LNCaP}$ cells. The average size of non-coated nanoparticle was approximately $120-130 \mathrm{~nm}$ with about $+50 \mathrm{mV}$ of $\zeta$-poten- 
A. PC-3 cells
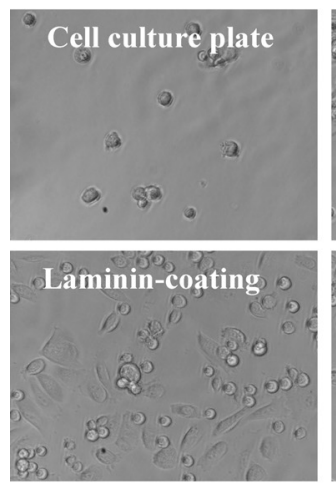

B. $\mathrm{LNCaP}$ cells
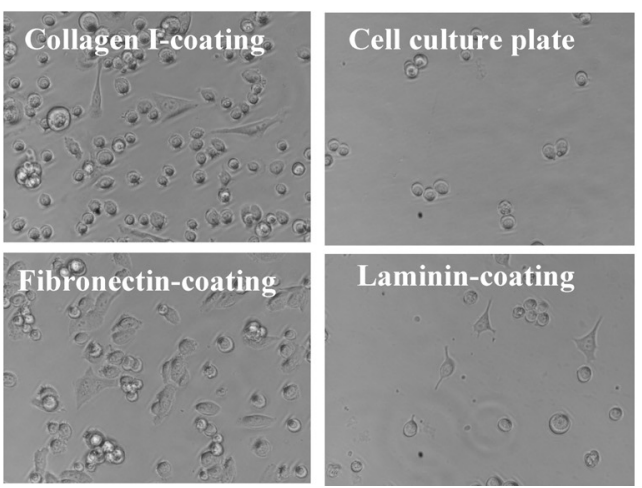

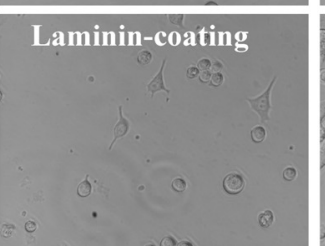

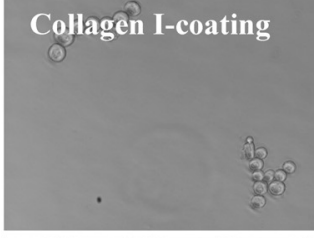

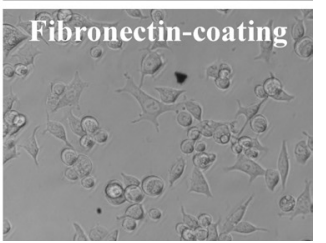

Fig. 1. Photomicrografts of PC-3 (A) and LNCaP (B) Plated on a Cell Culture Plate Coated with ECM Protein Cells were incubated for $1 \mathrm{~h}$ with non-coated, Fn-, Col- or Lam-coated tissue culture palates. Magnification $\times 200$.

Table 1. Differential Expression of Genes in PC-3 and LNCaP Cells

\begin{tabular}{lcc}
\hline \hline Gene name & PC-3 & LNCaP \\
\hline Meth & + & +++ \\
CD44 & +++ & - \\
E-cadherin & + & ++ \\
CEA & + & +++ \\
Catenin $\beta 1$ & +++ & +++ \\
Catenin delta1 & + & +++ \\
Cathepsin L & + & + \\
Fibronectin 1 & + & + \\
Integrin $\alpha 2$ & + & ++ \\
Integrin $\beta 1$ & ++ & +++ \\
Hyaluronidases & ++ & - \\
PECAM-1 & ++ & + \\
Maspin & ++ &
\end{tabular}

This table shows the optical density of the spot in cDNA array. Median pixel intensity of each spot in cDNA array was calculated using ScanAlyze software, which is a program for DNA microarray imaging and extracts median pixel intensity (range: 0 65536) from the image data of spots. +++ : pixel intensity $>25000,++: 25000$ $15000,+: 15000-5000,-:<5000$.

tial, and the size of non-coated nanoplex mixed with $2 \mu \mathrm{g}$ plasmid DNA and the nanoparticle increased to $800 \mathrm{~nm}$ (about $+39 \mathrm{mV}$ of $\zeta$-potential). In ECM protein-coated nanoplexes, their size depended on ECM protein. When the nanoplex of $2 \mu \mathrm{g}$ plasmid DNA was complexed with $2 \mu \mathrm{g}$ of Fn and Col, the sizes of Fn and Col nanoplexes decreased to approximately 230 and $430 \mathrm{~nm}$, respectively, and their $\zeta$-potential decreased to -16.3 and $+8.0 \mathrm{mV}$, respectively, suggested $\mathrm{Fn}$ and $\mathrm{Col}$ coating on nanoplexes. In contrast, when the nanoplex of $2 \mu \mathrm{g}$ plasmid DNA was complexed with $2 \mu \mathrm{g}$ of Lam, the size of the Lam nanoplex was greatly increased in size $(11 \mu \mathrm{m})$ and $\zeta$-potential was decreased to $+14.2 \mathrm{mV}$. It was not clear why the size of the nanoplex decreased after mixing with $\mathrm{Fn}$ and Col.

We examined the effect of ECM proteins on the transfection mediated by the nanoplexes. Enhanced transfection was observed when $2 \mu \mathrm{g}$ of Fn and Lam was associated with nanoplexes of $2 \mu \mathrm{g}$ plasmid DNA (Fig. 2A). Two and five micrograms of these proteins showed a similar level of luciferase gene expression, indicating that $2 \mu \mathrm{g}$ of ECM proteins were sufficient for transfection. In LNCaP cells, transfection efficiency was slightly increased by coating with Fn or Col, but not significantly compared to the non-coated nanoplex (Fig. 2B). Fn, Col and Lam nanoplexes exhibited slightly decrease of cell viabilities $(74,86$ and $85 \%$, respectively, compared with that of non-coated nanoplex (89\%)) (data not shown). Many parameters including size of nanoplex are known to affect transfection efficiency. ${ }^{18,19)}$ Large lipoplexes over $700 \mathrm{~nm}$ in mean diameter induced efficient transfection than lipoplexes with $250 \mathrm{~nm}{ }^{20)}$ The size of Lam nanoplex $(11 \mu \mathrm{m})$ was greatly larger than that of Fn and Col nanoplexes; therefore, Lam nanoplex might increase gene transfer efficiency in PC-3 cells.

Our results demonstrated that the association of Fn with cationic nanoplex produced a ternary complex, which showed much higher transfection activity into PC-3 cells. Fn is a large $(440 \mathrm{kDa})$ multidomain extracellular matrix protein with an RGD site that is not exposed in its native compact conformation. Fn adopts a conformation that is more open than its compact conformation when bound to liposome vector. ${ }^{21)}$ This open confirmation exposes a hidden RGD site in Fn that stimulates binding to adhesion molecules. ${ }^{21)}$ In our study, the hidden RGD site of the Fn nanoplex might be exposed. CD44 expression was seen in PC-3 cells but not LNCaP cells. CD44 can bind to Col, Fn and Lam, ${ }^{22)}$ and the ligands of integrin $\alpha 2 \beta 1$ are Lam and Col. ${ }^{23)}$ This suggested that Fn nanoplex was likely to bind mainly to CD44, and to increase the association of nanoplex with the cells, resulting in enhanced transfection efficiency in PC-3 cells.

Among ECM-coated nanoplexes, the Fn nanoplex $(2 \mu \mathrm{g}$ Fn for $2 \mu \mathrm{g}$ plasmid DNA) was injectable in size $(230 \mathrm{~nm})$ and increased transfection efficiency in PC-3 cells, being comparable to lipofectamine 2000. Therefore, we decided to use Fn nanoplex for in vivo gene delivery by direct injection into PC-3 tumor xenografts. As a control, we used commercially available transfection polymer in vivo jetPEI, because non-coated nanoplex was not injectable in size. To analyze the level of luciferase expression, we imaged mice and quantified bioluminescence using an NightOWL LB981 NC100 system, expressed as photon/s/tumor. Figure 3 shows the efficiency of Fn nanoplex (10 $\mu \mathrm{g}$ Fn for $10 \mu \mathrm{g}$ plasmid DNA) to carry DNA into PC-3 tumor xenografts in nude mice. Fn nanoplex showed significantly 4-fold more efficient transfection activity than in vivo jetPEI (Figs. 3A, B). This finding suggested that Fn proteins with exposed conformation could effectively interact with the corresponding receptors, allowing the strong induction of expression in metastatic prostate tumor. Considering the simplicity, level of efficiency, and 

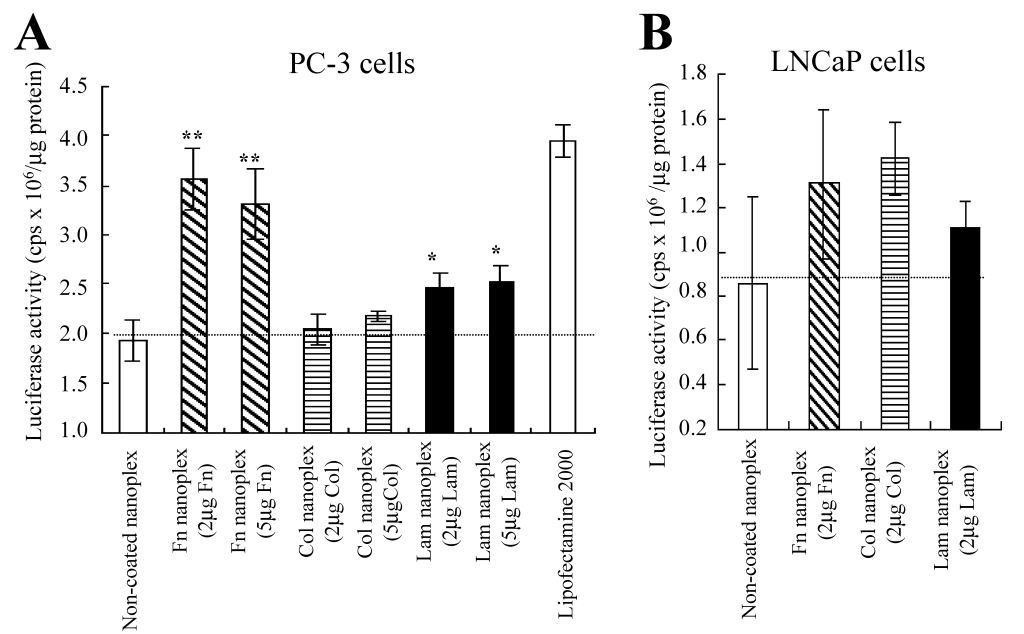

Fig. 2. The Effect of ECM Protein Complexed with Nanoplex on Luciferase Gene Expression in PC-3 (A) and LNCaP (B) Cells The nanoplex of $2 \mu \mathrm{g}$ plasmid DNA was complexed with $2 \mu \mathrm{g}$ or $5 \mu \mathrm{g}$ of Fn, Col or Lam. $* p<0.05$, $* * p<0.01$ compared with non-coated nanoplex.
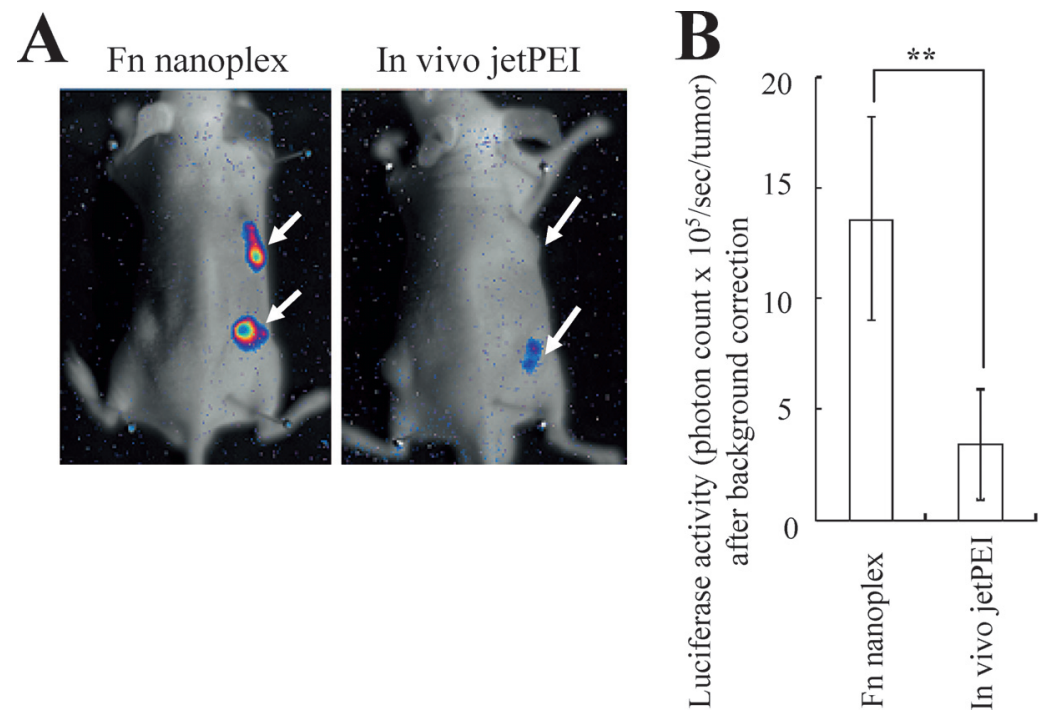

Fig. 3. Tumor Transfection in Vivo

Xenografts of PC-3 tumor cells were directly injected with $10 \mu \mathrm{g}$ plasmid DNA by Fn nanoplex (Fn $10 \mu \mathrm{g}$ ) and in vivo jetPEI polyplex, respectively. Arrowheads indicate solid tumor injected DNA into xenografts. Twenty-four hours after i.t injections, mice were imaged and bioluminescence was quantified. A shows pseudocolor luminescent images from blue (least intense) to red (most intense), representing light emitted from tumors superimposed over a grayscale reference image of representative mice from each group of three. B, Quantification of emitted photons from each tumor. $* * p<0.01$ compared with Fn nanoplex.

minimal cost, this technique emerges as a valuable tool for gene delivery.

In conclusion, we focused on the alternation of ECM and adhesion molecules in the progression of prostate cancer, and found that Fn-coated nanoplex increased transfection efficiency in metastatic prostate tumor. These results indicated that extracellular matrix-coated nanoplex could facilitate the efficient transfection of prostate tumor cells.

Acknowledgements This project was supported in part by a grant from the Promotion and Mutual Aid Corporation for Private Schools of Japan, and by a Grant-in-Aid for Scientific Research from the Ministry of Education, Culture, Sports, Science and Technology of Japan.

\section{REFERENCES}

1) Scott G., Cassidy L., Busacco, A., J. Invest Dermatol., 108, 147-153 (1997).

2) Saiki I., Jpn. J. Pharmacol., 75, 215-242 (1997).

3) Romanov V. I., Goligorsky M. S., Prostate, 39, 108-118 (1999).

4) Anwer K., Kao G., Rolland A., Driessen W. H., Sullivan S. M., J. Drug Target, 12, 215-221 (2004).

5) Harvie P., Dutzar B., Galbraith T., Cudmore S., O’Mahony D., Anklesaria P., Paul R., J. Liposome Res., 13, 231-247 (2003).

6) Schiffelers R. M., Ansari A., Xu J., Zhou Q., Tang Q., Storm G., Molema G., Lu P. Y., Scaria P. V., Woodle M. C., Nucleic Acids Res., 32, e149 (2004).

7) Kunath K., Merdan T., Hegener O., Haberlein H., Kissel T., J. Gene Med., 5, 588-599 (2003).

8) Jenkins R. G., Meng Q. H., Hodges R. J., Lee L. K., Bottoms S. E., Laurent G. J., Willis D., Ayazi S. P., McAnulty R. J., Hart S. L., Gene Ther., 10, 1026-1034 (2003).

9) Hart S. L., Arancibia-Carcamo C. V., Wolfert M. A., Mailhos C., O’Reilly N. J., Ali R. R., Coutelle C., George A. J., Harbottle R. P., 
Knight A. M., Larkin D. F., Levinsky R. J., Seymour L. W., Thrasher A. J., Kinnon C., Hum. Gene Ther, 9, 575-585 (1998).

10) Chowdhury E. H., Nagaoka M., Ogiwara K., Zohra F. T., Kutsuzawa K., Tada S., Kitamura C., Akaike T., Biochemistry, 44, 12273-12278 (2005).

11) Hattori Y., Kubo H., Higashiyama K., Maitani Y., J. Biomed. Nanotech., 1, 176-184 (2005).

12) Igarashi S., Hattori Y., Maitani Y., J. Control Release, 112, 362-368 (2006).

13) Hattori Y., Maitani Y., Cancer Gene Ther, 12, 796-809 (2005).

14) Hattori Y., Maitani Y., Cancer Sci., 97, 787-798 (2006).

15) Aalinkeel R., Nair M. P., Sufrin G., Mahajan S. D., Chadha K. C., Chawda R. P., Schwartz S. A., Cancer Res., 64, 5311-5321 (2004).

16) Dhir R., Gau J. T., Krill D., Bastacky S., Bahnson R. R., Cooper D. L.,
Becich M. J., Mol. Diagn., 2, 197-204 (1997).

17) Kostenuik P. J., Sanchez-Sweatman O., Orr F. W., Singh G., Clin. Exp. Metastasis, 14, 19-26 (1996).

18) Almofti M. R., Harashima H., Shinohara Y., Almofti A., Li W., Kiwada H., Mol. Membr. Biol., 20, 35-43 (2003).

19) Escriou V., Ciolina C., Lacroix F., Byk G., Scherman D., Wils P., Biochim. Biophys. Acta, 1368, 276-288 (1998).

20) Turek J., Dubertret C., Jaslin G., Antonakis K., Scherman D., Pitard B., J. Gene Med., 2, 32-40 (2000).

21) Halter M., Antia M., Vogel V., J. Control Release, 101, 209-222 (2005).

22) Goodison S., Urquidi V., Tarin D., Mol. Pathol., 52, 189-196 (1999).

23) Fornaro M., Manes T., Languino L. R., Cancer Metastasis Rev., 20, $321-331(2001)$. 